\title{
Study on a compression algorithm of surveillance video based on
}

\section{Fourier-Mellin transformation}

\author{
Yawen Li \\ Electronic information and electrical college of engineering, Shangluo University, Shangluo, \\ 726000,China; \\ slxylyw@163.com
}

Keywords: Surveillance video;Fourier-Mellin;Constant geometric dimension;Movement parameters Abstract. The compression algorithm of surveillance video files was proposed based on fourier-mellin transformation.In the paper, the coefficient of Fourier-Mellin were achieved to vectorization with the new algorithm,the surveillance video contains contiguous image frames, three groups of movement parameters were extracted before transmission depend on the constant geometric dimension, including the translation, rotation and scaling, it can more better to restore the original .This compression algorithm after supervising and controling video, parameter packet and reference were only transmitted in process, therefore, it was being useful to overcome a question of the transmission redundancy information in traditional compression video leading to the characteristics of speed slowly. The experiment result expresses that the algorithm is viable, the small efficient calculation and the high compression ratio.

\section{Introduction}

With the accelerating pace of life, people are increasingly concerned about the safety of the surrounding, monitoring equipment has been setted relatively in common, such as: parking surveillance, living community public security monitoring and ATM monitoring picture ${ }^{[1]}$, etc. It can timely warn dangerous events and reduce the economic loss through the staff to view the surveillance video, however, the key to video compression is to reduce and remove redundant information $^{[2]}$ in the image frame or interframe. In this paper, A transformational surveillance video compression algorithm based on Fourier-Mellin is proposed that can send and store data, implement efficient real-time transmission of video files, allows the user to discover in time the abnormal situation and nip in the bud.

\section{The algorithm thought of Fourier-Mellin}

The displacement transformation

The original reference image corresponds to the data matrix as $f_{1}(x, y)$, image matrix of $f_{2}(x, y)$ respective the data matrix ${ }^{[2]}$ that $f_{1}(x, y)$ takes $\mathrm{x}$ and y direction respectively translate $\mathrm{x}_{0}$ and $\mathrm{y}_{0}$.

$$
f_{2}(x, y)=f_{1}\left(x-x_{0}, y-y_{0}\right)
$$

If $\mathrm{f}_{1}$ and $\mathrm{f}_{2}$ correspond to Fourier transform respectively as $\boldsymbol{F}_{\mathbf{1}}(\boldsymbol{u}, \boldsymbol{v})$ and $\boldsymbol{F}_{\mathbf{2}}(\boldsymbol{u}, \boldsymbol{v})$.It has the following relationship ${ }^{[3]}$ between them: 


$$
F_{2}(u, v)=F_{1}(u, v) e^{-j\left(u x_{0}+v y_{0}\right)}
$$

(2) Geometry scaling invariance of image

It includes translation,scale invariance and Rotation invariance ${ }^{[4]}$.

About translation and scale invariance, use the normalized algorithm introduced in Zemike moment to take normalized image into a new image:

$$
f_{2}(x, y)=f(x / \lambda+\bar{x}, y / \lambda+\bar{y})
$$

When the orthogonal Fourier-Mellin used for image recognition, setting the image do rotation of the angle of the $\zeta$, after rotation the image is $f(r, \theta)=f(r, \theta-\zeta)^{[5]}$, Calculating orthogonal after the rotated image Fourier-Mellin ${ }^{[6]}$.

$$
\phi_{m n}^{\prime}=\frac{1}{2 \pi a} \int_{0}^{2 \pi} \int_{0}^{1} Q_{n}(r) e^{-i m \theta} f(r, \theta-\zeta) r d r d \theta
$$

If $\theta^{\prime}=\theta-\zeta$,

then

$$
\phi_{m n}^{\prime}=\frac{1}{2 \pi a} \int_{0}^{2 \pi} \int_{0}^{1} Q_{n}(r) e^{-i m \theta} f\left(r, \theta^{\prime}\right) r d r d \theta^{\prime}
$$

$$
=\left[\frac{1}{2 \pi a} \int_{0}^{2 \pi} \int_{0}^{1} Q_{n}(r) e^{-i m \theta} f\left(r, \theta^{\prime}\right) r d r d \theta^{\prime}\right] e^{-i m \zeta}=\phi_{m n} e^{-i m \zeta}
$$

The model of orthogonal Fourier-Mellin moments ${ }^{[6]}$ remains unchanged, only varying a corresponding phase. Thus, Orthogonal Fourier-Mellin has rotation invariance ${ }^{[6-7]}$.The coefficient of Fourier-Mellin transform was extracted and vectorization according to image's the geometric scale parameter invariant, including the corresponding motion parameters, Figure1 was showed the invariant geometric feature based on Fourier-Mellin transform.

\section{The extraction of motion parameters}

Base on Fourier-Mellin transformation to parameters extraction,firstly,translation parameters were extracted; then, the rotation and scaling parameters has been extracted with Fourier-Mellin in frequency domain. The amplitude spectrum of frequency domain was converted to polar coordinates and logarithmic coordinates, it was found that the method of solving rotation and scaling parameters actually transformation to be logarithm-polar coordinates domain ${ }^{[8]}$ after transformation, then to solve the problem of translation amount. Figure 2 was shown the concrete steps of monitor video compression programs .

\section{Simulation result}

A monitoring video file of phone.avi was studied in the experiment, figure 3 shows more frames of monitoring video and figure4 expresses extraction of motion parameter in GUI. Firstly, we will resolve the object file to multi-frame, the first frame image is selected as a reference frame with extracting the motion parameters between consecutive frames. If the change is not obvious between consecutive frames, and choose to the interval of a few frames, then extracting and storing the motion parameters into data.mat file. It only transmited the reference frame of ref.jpg 
and data.mat files in transformation, it can recover the original image frame base on the reference frame and motion parameters informations in terminal, which is connected the video file and named test.avi. In order to apply widely, the process of detection and recovery encapsulation for executing .exe file, the experiment named hfavi.exe file, it will also get the recovered video file.

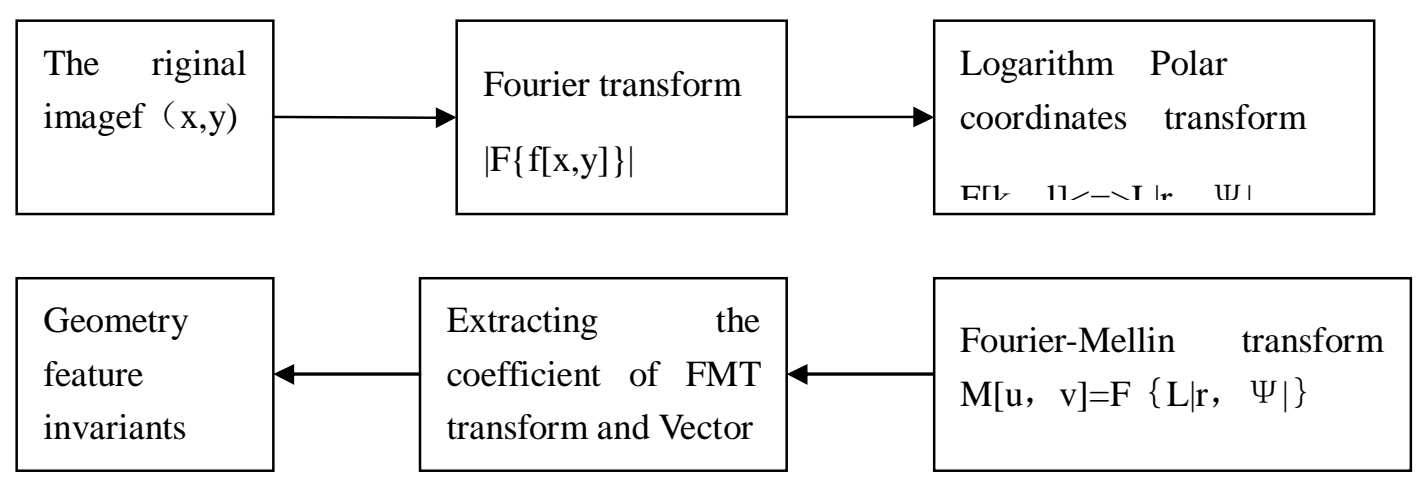

Fig.1 Invariant geometric feature of Fourier-Mellin transform

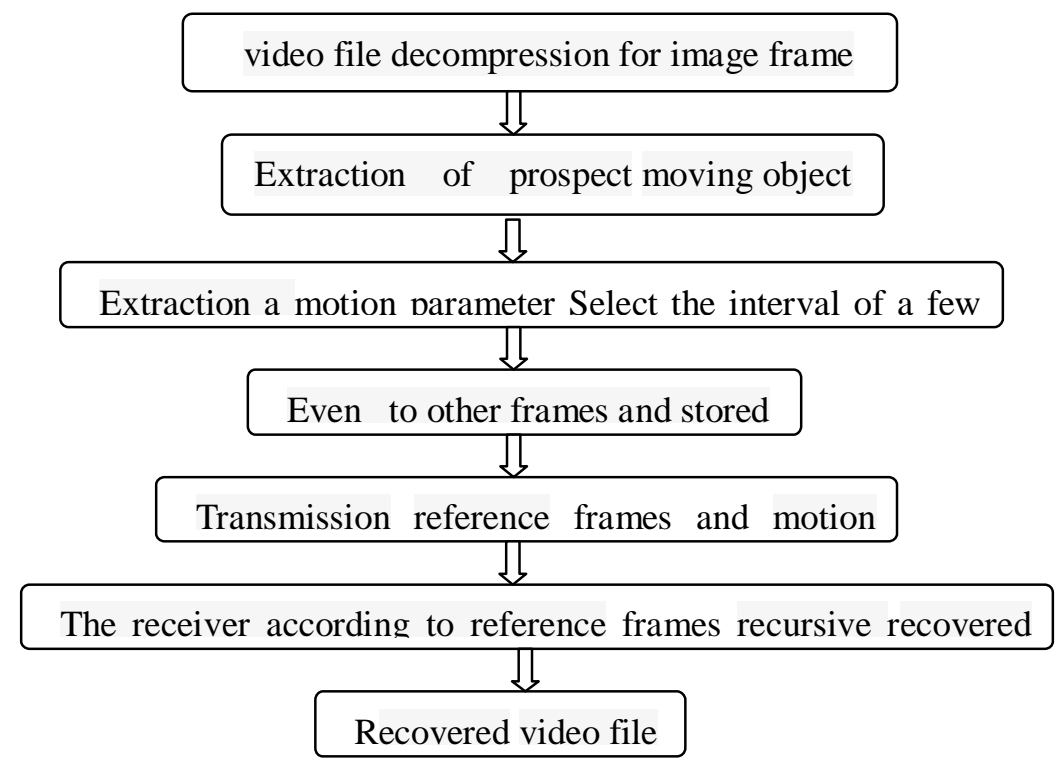

Fig. 2 concrete steps of monitor video compression

\section{Conclusion}

The extraction of surveillance video file compression and recovery algorithm based on the motion parameters is proposed in this paper, the experimental results showed that the algorithm can fast extract the motion parameters of adjacent image frames in a video file, and can better restore image frames, and then get the original video file. The algorithm compress background target simple video, especially for indoor monitor, ATM monitoring, parking lot monitor, etc., which background information for a long time is not to change of the surveillance video, extraction of motion parameters is more precise, less error and high quality, the advantage of compression and recovery is more significant. 


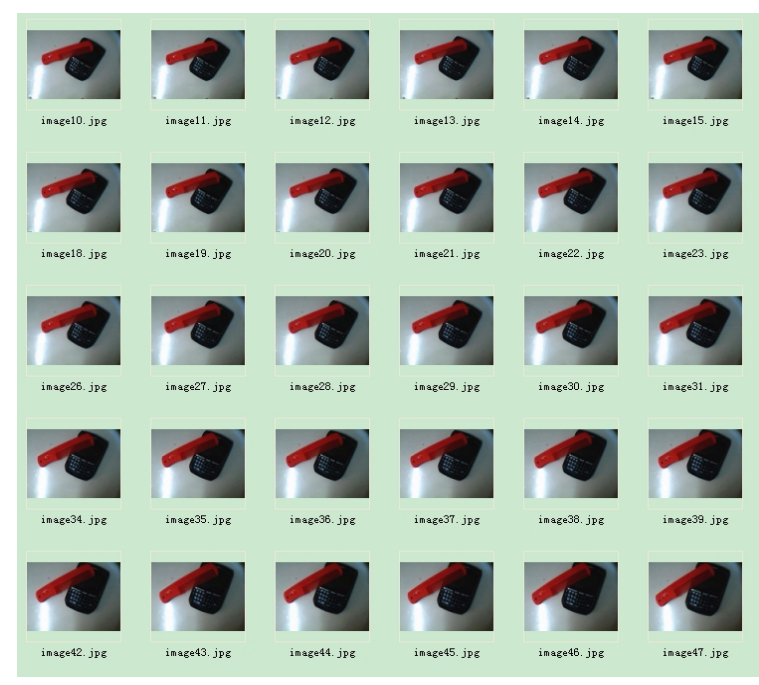

Fig.3 more frames of monitoring video

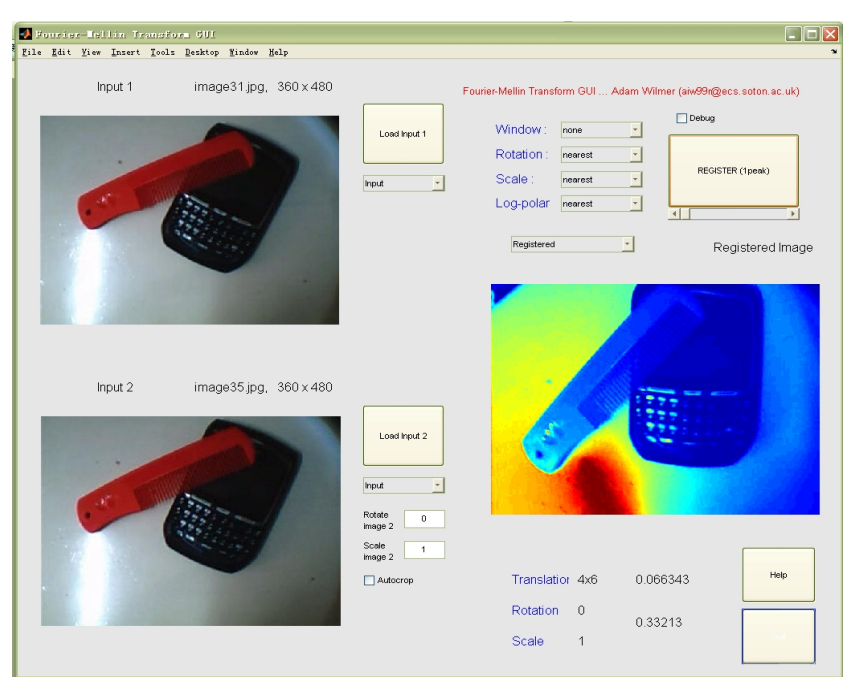

Fig.4 extraction of motion parameter in GUI

\section{Acknowledgements}

This work was supported by the scientific project of Shangluo University under Grant( 14SKY001).

\section{Reference}

[1] Jensen R, Shen Q:Fuzzy2rough sets assisted attribute selection , IEEE Transactions on Fuzzy Systems, Vol.1(2007), p:73-82.

[2] Lian Cai, Sidan Du, Duntang Gao: Journal of Electronics (china), Vol.5 (2005),p:300-306.

[3] Chunhavittayatera,S.Chitsobhuk, O.Tongprasert,:Image.Registration using Hough Transform and Phase Correlation, Advanced Communication Technology.The 8th International Conference, Phoenix Park, Vol.20-22 (2006),p:449-453.

[4] Onishi H,Suzuki H:Detection of rotation and parallel translation using Hough and Fourier transforms,Image Processing, Proceedings International Conference, San Diego, CA,(2008).

[5] Lucchese L,Vortelazzo G M,Monti C:High resolution estimation of planar rotations based on Fourier transform and radial projections, Circuits and Systems,2005,ISCAS, Proceedings of IEEE International Symposium, Hong Kong,China,(2005),p:546-552.

[6] R.Mukundan,S.H.Ong,P.A.Lee,Image analysis by Tchebichef moments,IEEE Trans.Image Processing Vol.9(2001,p:1357-1364.

[7] A.De Sena,D.Rocchesso,A fast mellin transform with applicantion in dafx,in:Proceedings of the 7th Internation Conference on Digital Audio Effects,Naples,Italy(2004).

[8] J.Flusser:On the indenpence of rotation moment invariants,Pattern Recongnition, Vol.35(2002), p:3015-3017. 